\title{
Propiedades psicométricas de la Medication Adherence Report Scale-Asthma en adultos asmáticos mexicanos ${ }^{1}$
}

\section{Psychometric properties of the Medication Adherence Report Scale-Asthma in asthmatic Mexicans adults}

\author{
Isaías Vicente Lugo González² y Cynthia Zaira Vega Valero ${ }^{3}$
}

Citación: Lugo G., I.V. y Vega V., C.Z. (2020). Propiedades psicométricas de la Medication Adherence Report Scale-Asthma en adultos asmáticos mexicanos. Psicología y Salud, 30(2), 275-285. https://doi.org/10.25009/pys.v30i2.2663.

\section{RESUMEN}

\begin{abstract}
El porcentaje de adherencia al medicamento de control en pacientes con asma es subóptimo. Cabe mencionar que tal proporción ayuda a identificar a los pacientes adherentes y no adherentes; sin embargo, esta medida no ofrece información sobre el patrón de uso del medicamento por parte del paciente, por lo cual existe la necesidad de disponer de una herramienta de evaluación que permita obtener tal dato. Así, el objetivo del presente trabajo fue evaluar las propiedades psicométricas de la versión en español de la Medication Adherence Report Scale-Asthma en pacientes mexicanos asmáticos, para lo cual se utilizó un muestreo por conveniencia. La muestra consistió en 280 adultos, quienes respondieron un cuestionario de datos sociodemográficos y clínicos y la citada escala. La muestra fue dividida en dos: en una mitad se realizó un estudio exploratorio, y en la otra uno confirmatorio de la escala. Los datos exploratorios muestran una escala unifactorial con siete ítems, con resultados estadísticamente aceptables. Se concluye que la MARS-A es un instrumento confiable y válido para la evaluación de conductas de adherencia al medicamento de control en adultos mexicanos con asma.
\end{abstract}

Palabras clave: Adherencia; Asma; Tratamiento de control; Confiabilidad; Validez.

\begin{abstract}
In general, the percentage of medication adherence in patients with asthma is sub-optimal. Such percentage allows for distinguishing adherent from not adherent patients. However, this gross measure does not provide information about of the patient pattern of medication. Thus, there is a need for assessment tools for this type of data collection. The aim of the present study was to evaluate the psychometric properties of the Spanish version of MARS-A in Mexican patients with asthma. Through convenience sampling recruitment, participants included 280 adults with asthma who answered sociodemographic and clinical questionnaires and the MARS-A. An exploratory analysis was carried out for half the participants, and a confirmatory analysis on the other half. Exploratory data showed a single factor scale with seven items and statistically acceptable results. It is concluded that the MARS-A is a reliable and valid instrument to assess medication adherence behaviors in Mexican adults suffering asthma.
\end{abstract}

Keywords: Adherence; Asthma; Treatment control; Reliability; Validity.

\footnotetext{
${ }^{1}$ Los autores agradecen al CONACyT por el financiamiento para los estudios del primer autor como tutorado de la segunda. Artículo recibido el 29 de marzo y aceptado el 9 de agosto de 2019.

${ }^{2}$ Doctorado en Psicología y Salud, Facultad de Estudios Superiores Iztacala, Universidad Nacional Autónoma de México, Av. De los Barrios s/n, Los Reyes Iztacala, 54090 Tlalnepantla, Edo. de México, México, tel. (55)56-23-12-94, correo electrónico: isaiasvlg@comunidad.unam.mx.

${ }^{3}$ Coordinación de Investigación y Posgrado, Facultad de Estudios Superiores Iztacala, Universidad Nacional Autónoma de México, Av. De los Barrios s/n, Los Reyes Iztacala. 54090 Tlalnepantla, Edo. de México, tel. (55)56-23-12-94, correo electrónico: czaira.vega@unam.mx.
} 


\section{INTRODUCCIÓN}

$\mathrm{E}$ 1 asma es una enfermedad crónica en la que se presenta inflamación de la vía aérea, obstrucción, sensibilidad e hiperreactividad (Global Initiative for Asthma [GINA], 2016). La evidencia refiere que en su desarrollo están implicados factores genéticos y medioambientales (Guía Mexicana de Asma [GUIMA], 2017). Se estima que más de 300 millones de personas la padecen en todo el mundo, de las cuales cerca de nueve millones residen en México, cifra que va en aumento (García, Fernández, Martínez, Franco y Pérez, 2012; Secretaría de Salud [SSA], 2016; World Health Organization [WHO], 2011). En los últimos años el asma se ubicó como uno de los cuatro padecimientos crónicos más atendidos en adultos, principalmente en los servicios de urgencias y hospitalización (Dirección General de Epidemiología [DGE], 2015; Instituto Nacional de Enfermedades Respiratorias [INER], 2018).

En el caso de este padecimiento, una continua atención hospitalaria refleja una falta de control normalmente asociada a una pobre adherencia al medicamento, lo que compromete la calidad de vida y afecta económica y emocionalmente al enfermo, incrementando el riesgo de crisis, nuevas hospitalizaciones y muerte (Ebmeier et al., 2017; Foster, Lavoie y Bouler, 2011; GINA, 2016; GUIMA; 2017).

Las conductas de adherencia son uno de los pilares principales del tratamiento para el asma (Engelkes, Janssens, de Jongste, Sturkenboom y Verhamme, 2015; GINA, 2016; WHO, 2003); sin embargo, se sabe que estos pacientes tienen porcentajes subóptimos de adherencia al tratamiento de control, los que oscilan entre 30 y 70\% (Bender y Bender, 2005; Fernández y Merino, 2015; Horne, 2011; Laforest et al., 2009), lo que es un factor de riesgo para que los tratamientos sean efectivos, ya que dicha efectividad disminuye catorce veces cuando los porcentajes de adherencia son menores a 80\% (Dunbar-Jacob, Schlenk y McCall, 2012).

Las anteriores cifras únicamente ofrecen una cuantificación del porcentaje de uso del tratamiento con base en un criterio temporal, con lo que se puede identificar qué personas son adherentes y cuales no, pero no ofrece evidencias sobre el patrón de uso que los pacientes hacen del tratamiento, lo que es importante en virtud de que la ad- herencia generalmente se define como un patrón conductual de uso del tratamiento que debe corresponder a las indicaciones médicas (DiMatteo, Haskard-Zolnierek y Martin, 2012; Dunbar-Jacob et al., 2012), lo que la sitúa como un fenómeno claramente psicológico (Rodríguez y Rentería, 2016).

De acuerdo con Dunbar-Jacob et al. (2012), el estudio de la adherencia se debe centrar en el patrón de adherencia o en la falta de la misma, y luego en su cuantificación, por lo que dichos autores refieren que hay al menos seis patrones de adherencia: 1) adoptar un régimen de tratamiento; 2) suspender tempranamente el tratamiento; 3) reducir el número de dosis del tratamiento; 4) usar excesivamente el tratamiento; 5) variar la frecuencia de uso del tratamiento; 6) hacer intervalos inadecuados en el uso del tratamiento; por lo que sugiere el empleo de herramientas que ofrezcan más información sobre tales patrones, además de su necesaria cuantificación.

En este contexto, es necesario señalar que hay diferentes métodos para la evaluación de la adherencia en el paciente con asma, normalmente clasificados en directos objetivos (análisis bioquímico, dispositivos electrónicos, evaluación de la técnica de inhalación), indirectos objetivos (conteo de dosis, datos farmacéuticos, juicio clínico) e indirectos subjetivos (autorreporte del paciente o familiar) (Lam y Fresco, 2015; López, Romero, Parra y Rojas, 2016). Ahora bien, de acuerdo con Foster et al. (2011) y Lam y Fresco (2015), en la elección del método de evaluación se debe tomar en cuenta su función, los recursos disponibles, las características de la población, los objetivos de la evaluación, el patrón de adherencia a evaluar y la definición de adherencia empleada. Por ello, el método más adecuado será aquel que implique menores costos, que sea fácilmente empleado e interpretado, y que tenga una mayor confiabilidad.

Derivado de lo anterior, se sabe que el empleo de instrumentos de autorreporte es el método más utilizado y recomendado en el contexto de la investigación psicológica en el campo de la salud debido a su bajo costo, gran eficacia, rápida aplicación y evaluación en términos conductuales. No obstante, presenta algunas limitaciones, como respuestas sujetas al olvido, inexactitud, sobrestimación, deseabilidad social y respuestas que describen el patrón de uso y el nivel de adherencia, 
mas no el momento y las condiciones exactas de su uso (Horne y Weinman, 1999; Foster et al., 2011; Lam y Fresco, 2015; Stirratt et al., 2015). Para minimizar esas limitaciones se recomienda que se garantice el anonimato, que las instrucciones y los ítems no sean amenazantes o que impliquen un juicio de valor negativo, y que se evalúe en términos de la falta de adherencia y no en qué tan adherente es el paciente (Horne y Weinman, 1999; Horne y Weinman, 2002; Mora et al., 2011; Stirratt et al., 2015).

Uno de los instrumentos de autorreporte sobre conductas de adherencia en pacientes con asma $\mathrm{y}$ otras enfermedades respiratorias que toma en cuenta las indicaciones antes referidas es la Medication Adherence Report Scale-Asthma (MARS-A, en lo sucesivo) (Horne y Hankins, 2002; García y Pérez, 2015). Esta escala un instrumento de autorreporte, con diez ítems para evaluar el patrón de falta de adherencia, nueve para medir conductas intencionales de falta de adherencia y uno más para valorar la adherencia no intencional (olvido).

En Estados Unidos, la MARS-A, tanto en inglés como en español, fue validada por Cohen et al. (2009) en pacientes con asma, obteniendo coeficientes alfa de Cronbach de 0.85 y 0.86 , respectivamente, una adecuada confiabilidad test-retest $(r=0.65, p \leq .001) \mathrm{y}$, por medio del análisis de componentes principales, un factor que explicó $88.7 \%$ de la varianza. Por otro lado, Mora et al. (2011) evaluaron la estabilidad temporal y la estructura factorial de la escala, identificando dos factores a los que denominaron "factor de falta de adherencia basado en cambios somáticos", y "factor de falta de adherencia intencional y evitación de la medicación". La escala mostró estabilidad temporal en cuatro momentos distintos (línea base, a un mes, a los tres meses y a los 12 meses), además de ser confiable con coeficientes alfa de 0.88 a 0.89 : el primer factor de 0.73 a 0.75 y el segundo de 0.83 a 0.85 .

La MARS-A ha correlacionado de manera adecuada con otros métodos para evaluar la adherencia en pacientes con asma; por ejemplo, los registros farmacéuticos de reabastecimiento de medicamentos, $(r=0.46, p \leq .001)$ (Menckeberg et al., 2008) y dispositivos de monitoreo electrónico $(r=0.42, p \leq .001$ [Cohen et al., 2009] y $r=0.53$, $p \leq .001$ [Ohm y Aaronson, 2006]). Además, su uso se ha extendido a diferentes países en estudios observacionales y de intervención (Champman et al., 2015; Emilsson et al., 2011; Horne y Weinman, 1999, 2002; Ovchinikova, Smith y Bosnic-Anticevich, 2011; Ponieman, Wisnivesky, Leventhal, Musumeci-Szabó y Halm, 2009; Sofianou et al., 2013; Wroe, 2002; Zwikker et al., 2014).

Finalmente, debe mencionarse que en la última década la MARS-A ha sido adaptada para pacientes con enfermedades crónicas (Alsous et al., 2017; Mahler et al., 2010), artritis reumatoide (Salt, Hall, Peden y Horne, 2012), usuarios de estatinas (Ladova et al., 2014), EPOC (Tommelein, Mehuys, Van Tongelen, Brusselle y Boussery, 2014), asma infantil (García, Brand, Kaptein y Klok, 2016), enfermedad de Crohn (Scribano et al., 2018), infarto (Lin et al., 2018) y dolor crónico (Sampaio, Azevedo, Dias, Horne y Castro, 2019); sin embargo, hasta hoy no se cuenta con datos psicométricos de confiabilidad y validez para México.

Derivado de lo anterior, este trabajo de tipo observacional y descriptivo -según la clasificación de Méndez, Namihira, Moreno y Sosa (2001)tuvo como propósito evaluar las propiedades psicométricas de la versión en español (ajustada) de la MARS-A en adultos mexicanos con asma.

\section{MÉTODO}

\section{Participantes}

Considerando un muestreo no probabilístico de sujetos voluntarios (Hernández, Fernández y Baptista, 2014), se obtuvo una muestra total de 280 pacientes con asma del Instituto Nacional de Enfermedades Respiratorias (INER) de la Ciudad de México (CDMX), de los cuales 209 (74.6\%) fueron mujeres y $71(25.4 \%)$ hombres, con un promedio de edad de 43.90 años (D.E. $=14.73$ ). Respecto del lugar de residencia, 183 (65.4\%) fueron de la CDMX, 72 (25.7\%) del Estado de México y 25 (8.9\%) de otros estados de la República Mexicana. La mayoría, 152 de ellos, estaban casados (50.3\%) y 81 vivían con una pareja (29.3\%); 74 (26.4\%) eran empleados, $32(11.4 \%)$ profesionistas y 93 $(32.9 \%)$ se dedicaban a otras actividades. En cuanto a su nivel educativo, $69(24.6 \%)$ contaban con licenciatura, 69 (24.6\%) con secundaria, 61 (21.8\%) con preparatoria, $37(13.2 \%)$ con carrera técnica y 44 (15.8) con otro nivel educativo. Finalmente, 
respecto a la enfermedad, el tiempo de evolución tuvo una media de 13.87 (D.E. $=12.60$ ).

Los criterios de inclusión necesarios para que los pacientes pudieran participar fueron los siguientes: ser mayor de edad; tener un diagnóstico confirmado de asma; contar con un tratamiento de control; aceptar participar en la investigación, y firmar un formato de consentimiento informado en el que se especificó la aprobación del proyecto por parte del Comité de Ética en Investigación del INER, con código C47-18. Los criterios de exclusión fueron ser menor de edad, no tener diagnóstico confirmado de asma, tener un diagnóstico de asma, pero no haber iniciado un tratamiento de control, y no aceptar participar en la investigación. Finalmente, los criterios de eliminación fueron el aceptar participar y después declinar, y responder los instrumentos de manera incompleta.

\section{Procedimiento}

Los pacientes que participaron en la investigación fueron captados en la sala de espera de la Clínica de Asma del INER. A cada paciente se le exponían individualmente las razones para haber sido abordado, se le explicaban los objetivos de la investigación y se le pedía su participación voluntaria para responder los instrumentos. Cuando devolvía los instrumentos, se revisaba que estuvieran respondidos en su totalidad. Finalmente, se le entregaba una copia del formato de consentimiento informado.

\section{Instrumentos}

\section{Cédula de datos sociodemográficos y clínicos}

Consistió en un conjunto de preguntas sobre los datos personales, familiares, educativos, y ocupacionales de los participantes, así como los antecedentes y severidad de enfermedad y el tipo de tratamiento.

\section{Medication Adherence Report Scale-Asthma (Horne y Hankins, 2002).}

Este instrumento evalúa el patrón de conductas de falta de adherencia al medicamento de control en pacientes con asma y está constituido por diez ítems, nueve de los cuales implican conductas in- tencionales de falta de adherencia y uno sobre el olvido para usar el tratamiento. Cuenta con un formato de respuesta tipo Likert de cinco puntos, que corresponden a "siempre", "casi siempre", "algunas veces", "de vez en cuando" y "nunca". El puntaje total de la escala se obtiene sumando las respuestas de cada ítem y dividiendo esa suma entre el número de ítems, por lo que puntajes más altos corresponden a un mejor nivel de adherencia. En este trabajo se definió el puntaje de 4.5 como punto de corte entre la no adherencia y la adherencia. El citado instrumento cuenta con datos adecuados de validez y confiabilidad, con un coeficiente alfa de Cronbach de .85. En este estudio únicamente se consideraron los nueve ítems correspondientes a la falta de adherencia intencional, sin tomar en cuenta el ítem correspondiente al olvido.

\section{Análisis de resultados}

Antes de la aplicación del instrumento se requirió efectuar algunos ajustes en la redacción de los ítems, el contenido de las respuestas y el número de las mismas tras llevar a cabo un piloteo con especialistas en medición y neumología, así como con pacientes asmáticos, cuyo propósito fue conservar o mejorar las propiedades psicométricas del instrumento (Reyes, 2011).

Dado que la muestra total del estudio estaba constituida por 280 pacientes, se decidió dividirlos en dos grupos para efectos de los análisis posteriores $(\mathrm{n} 1=100$ y $\mathrm{n} 2=280)$. En el análisis inicial se tomó en cuenta el número de ítems de la MARS-A (nueve) y se consideró una muestra de cien pacientes (n1), superando así el criterio de Nunnally y Bernstein (1994) de disponer de cinco participantes por ítem. Se utilizó el programa estadístico SPSS, versión 20 de Windows, realizando el siguiente proceso: 1) análisis descriptivos de las características de los pacientes; 2) evaluación de la normalidad de los datos de la escala, para así determinar el tipo de estadísticos a utilizar en el análisis psicométrico con base en el tamaño de la muestra y el programa empleado para el análisis de datos; 3) análisis de la frecuencia y sesgo de los ítems y la discriminación de reactivos para grupos extremos (percentiles uno y cuatro) para así determinar el comportamiento de los ítems de la 
escala; 4) correlación entre ítems y prueba total (considerando los ítems con correlaciones $>.20$ ) y el coeficiente alfa de Cronbach $(\alpha)$ para evaluar la consistencia interna; 5) evaluación de la estructura del instrumento mediante el análisis factorial exploratorio con el método de ejes principales con rotación promax; 6 ) comprobación de la pertinencia del modelo factorial mediante el índice Kaiser-Meyer-Olkin (KMO), tomando en cuenta datos $\geq .8$ y la significancia estadística de $X^{2}$ en la prueba de esfericidad de Bartlett, y 7) determinar el número de factores considerando los autovalores $\geq 1$ y la agrupación de los ítems a estos tomando en cuenta el criterio de $\geq .4$ (Ferrando y Anguiano, 2010; Pedrosa, Juarros, Robles, Basteiro y García, 2015; Reyes y García, 2008).

Hecho lo anterior, ya contado con el modelo factorial de la MARS-A, se comprobó empleando el paquete estadístico AMOS 24 de Windows y considerando una muestra de 180 pacientes (n2), cumpliendo así con el criterio de al menos veinte participantes por ítem, empleando para ello el método de estimación de máxima verosimilitud con la corrección robusta de Browne (Browne's Asymptptic Distribution Free, ADF) (Kline, 2011).

Para evaluar la bondad de ajuste del modelo se tomaron en cuenta los siguientes indicadores de ajuste absoluto: prueba de bondad de ajuste $X^{2}$ (no significativa para un buen ajuste) (Morata, Holgado, Barbero y Méndez, 2015); cociente resultante de $X^{2} / g l$ (cercano a 2 para un ajuste aceptable) (Kline, 2011); error cuadrático medio de aproximación (RMSEA) $<.05$, que implica un ajuste óptimo (valores entre $.05 \mathrm{y} .08$ implican un ajuste aceptable) (Browne y Cudeck, 1993) y residuo cuadrático medio $(\mathrm{RMR})<.05$. Se consideraron también los siguientes indicadores de bondad de ajuste general: indicador de ajuste general gama (GFI) e indicador de ajuste general ajustado (AGFI), ambos con datos de $\geq .95$ para un ajuste óptimo. Además, se tomaron en cuenta los siguientes indicadores de ajuste comparativo: índice de Tucker-Lewis (TLI) e índice de ajuste normado (NFI), en los que en ambos se esperarían valores $\geq$ de .9 a .95 para un ajuste satisfactorio (Kline, 2011). Finalmente, la validez convergente se comprobó a través de la varianza media extraída (AVE), la cual debe ser superior a 0.50 para determinar que más de la mitad de la varianza del constructo se explica por sus indicadores (Farrell, 2010).

\section{RESULTADOS}

\section{Validez de contenido de los ítems}

Los principales ajustes al instrumento se hicieron con el propósito de tener una mayor claridad; por ejemplo, el 1) ítem inicial: "Uso el medicamento de control menos veces de lo indicado" se ajustó como sigue: "Uso mi medicamento de control menos veces de lo que me indicó el doctor". Por su parte, las opciones de respuestas se modificaron y se les incluyó la frase "lo hago asi", ya que la forma de respuesta inicial ("siempre", "casi siempre", "pocas veces" y "nunca") era poco clara para los pacientes, quedando de la siguiente manera: "Siempre lo hago así", "Casi siempre lo hago así", "Pocas veces lo hago así" y "Nunca lo hago así". Además, las opciones se redujeron a cuatro para evitar respuestas centrales respecto de las opciones originales (DeVellis, 2012; Reyes, 1993, 2011), tomando en cuenta las recomendaciones para el desarrollo de instrumentos en México, e identificadas como una forma de respuesta común en este contexto (Reyes, 1993, 2011).

\section{MARS-A: estudio exploratorio}

Los datos referentes al comportamiento de los ítems de la MARS-A se muestran en la Tabla 1. Como puede observarse, cada una de las opciones de repuesta de los ítems fue elegida y el puntaje promedio para cada ítem fue alto (opciones tres y cuatro), lo que corresponde a puntajes altos en adherencia. El sesgo, la simetría y el resultado del estadístico W de Shapiro-Wilk corroboran su no normalidad, lo que justifica el empleo del estadístico U de Mann-Whitney para comprobar que cada ítem diferencia entre puntajes altos y bajos. Finalmente, en las correlaciones elemento-total corregidas se identificó que ninguno de los ítems estuvo por debajo de .20 , criterio para que cada ítem pueda ser considerado en los análisis subsecuentes. Sin embargo, es necesario notar que los ítems con correlaciones más bajas fueron el 7 y el 8, este último con una correlación de .262 . 
Tabla 1. Compartimento estadístico de la MARS-A.

\begin{tabular}{|c|c|c|c|c|c|c|c|c|c|c|c|}
\hline \multirow{2}{*}{ ÍTEMS } & \multicolumn{4}{|c|}{ Frecuencias } & \multicolumn{7}{|c|}{ Sesgo, normalidad, discriminación y correlación } \\
\hline & 1 & 2 & 3 & 4 & M & D.E. & $\mathbf{S}$ & $\mathbf{C}$ & $\mathbf{W}$ & $\mathbf{Z}$ & $\mathbf{C E}$ \\
\hline $\begin{array}{l}\text { 1. Uso mi medicamento de control } \\
\text { solo cuando lo necesito. }\end{array}$ & 25 & 7 & 19 & 49 & 2.92 & 1.25 & -6.33 & -1.30 & $.742 * *$ & $-6.17 * *$ & .512 \\
\hline $\begin{array}{l}\text { 2. Uso mi medicamento de control } \\
\text { solo cuando me falta el aire. }\end{array}$ & 20 & 7 & 17 & 56 & 3.09 & 1.19 & -.895 & -.857 & $.710^{* *}$ & $-5.83 * *$ & 661 \\
\hline $\begin{array}{l}\text { 3. Decido cuándo no usar mi medicamento } \\
\text { de control. }\end{array}$ & 12 & 5 & 21 & 62 & 3.33 & 1.02 & -1.39 & -.615 & $.668 * *$ & $-5.40 * *$ & .684 \\
\hline $\begin{array}{l}\text { 4. Trato de no usar mi medicamento } \\
\text { de control. }\end{array}$ & 16 & 3 & 18 & 63 & 3.28 & 1.11 & -1.29 & .131 & $.651^{* *}$ & $-5.60 * *$ & .602 \\
\hline $\begin{array}{l}\text { 5. Cambio el número de inhalaciones } \\
\text { de mi medicamento de control. }\end{array}$ & 4 & 4 & 16 & 76 & 3.64 & .746 & -2.28 & 4.79 & $.542 * *$ & $-3.62 * *$ & .490 \\
\hline $\begin{array}{l}\text { 6. Dejo de usar mi medicamento } \\
\text { de control por un tiempo cuando estoy } \\
\text { mejor. }\end{array}$ & 15 & 3 & 30 & 52 & 3.19 & 1.06 & -1.16 & .073 & $.717 * *$ & $-5.80 * *$ & .676 \\
\hline $\begin{array}{l}\text { 7. Uso mi medicamento de control } \\
\text { si mi tratamiento de rescate no funciona. }\end{array}$ & 12 & 4 & 22 & 62 & 3.34 & 1.01 & -1.43 & 3.23 & $.663 * *$ & $-4.69 * *$ & .415 \\
\hline $\begin{array}{l}\text { 8. Uso mi medicamento de control } \\
\text { antes de hacer algo que me provoque } \\
\text { dificultad para respirar. }\end{array}$ & 16 & 6 & 17 & 61 & 3.23 & 1.12 & -1.15 & 3.49 & $.678 * *$ & $-4.51 * *$ & .262 \\
\hline $\begin{array}{l}\text { 9. Uso mi medicamento de control } \\
\text { menos veces de lo que me indicó el doctor. }\end{array}$ & 6 & 4 & 25 & 65 & 3.49 & .835 & -1.77 & 2.56 & $.641 * *$ & $-5.23 * *$ & .602 \\
\hline
\end{tabular}

Nota: 1. "Siempre lo hago así", 2. "Casi siempre lo hago así”, 3. "Pocas veces lo hago así", 4. "Nunca lo hago así"; S: Simetría; C: Curtosis; W: Estadístico Shapiro-Wilk; z: Estadístico $U$ de Mann-Whitney; ** $p<0.01$; CE: Correlación elemento-total corregida.

\section{Análisis de confiabilidad y validez}

Los resultados en torno a la confiabilidad y validez de la MARS-A se muestran en la Tabla 2. En el primer análisis de la MARS-A se obtuvo un coeficiente $\alpha$ de 0.830 , con nueve ítems organizados en tres factores, KMO de $0.803 X^{2}$ de 330.988 $(\mathrm{p}=.000)$ y una varianza total explicada (VTE) de $54.57 \%$; sin embargo, el ítem 7 tuvo una carga factorial de .377 , por lo que fue eliminado y se corrió un siguiente análisis, en el cual se obtuvo un coeficiente $\alpha$ de 0.825 , con ocho ítems divididos en dos factores, KMO de $0.806, X^{2}$ de 330.298 $(p=.000)$ y VTE de $51.16 \%$; en este caso, el ítem 8 tuvo una carga factorial de .247 por lo que fue asimismo eliminado. En un siguiente análisis, el coeficiente $\alpha$ fue de 0.850 , con siete ítems divididos en dos factores (un indicador con dos ítems), KMO de $0.809, X^{2}$ de $293.180(p=.000)$ y VTE de $57.68 \%$. Finalmente, dada la estructura original del instrumento, se decidió forzar el análisis a un factor, considerando el indicador de dos ítems y los gráficos de sedimentación, los cuales mostraban un único factor, análisis que se muestra en la Tabla 2.
Tabla 2. Datos de confiabilidad y validez de la MARS-A.

\begin{tabular}{|l|c|}
\hline \multicolumn{1}{|c|}{ Ítem } & Carga \\
\hline $\begin{array}{l}\text { 3. Decido cuándo no usar mi medicamento } \\
\text { de control. }\end{array}$ & .810 \\
\hline $\begin{array}{l}\text { 6. Dejo de usar mi medicamento de control } \\
\text { por un tiempo cuando estoy mejor. }\end{array}$ & .785 \\
\hline $\begin{array}{l}\text { 4. Trato de no usar mi medicamento } \\
\text { de control. }\end{array}$ & .720 \\
\hline $\begin{array}{l}\text { 2. Uso mi medicamento de control solo } \\
\text { cuando me falta el aire. }\end{array}$ & .697 \\
\hline $\begin{array}{l}\text { 9. Uso mi medicamento de control menos } \\
\text { veces de lo que me indicó el doctor. }\end{array}$ & .644 \\
\hline $\begin{array}{l}\text { 5. Cambio el número de inhalaciones } \\
\text { de mi medicamento de control. }\end{array}$ & .540 \\
\hline $\begin{array}{l}\text { 1. Uso mi medicamento de control solo } \\
\text { cuando lo necesito. }\end{array}$ & .539 \\
\hline Alfa & 0.850 \\
\hline Autovalor & 3.772 \\
\hline KMO & 0.809 \\
\hline$X^{2}$ & $293.180 *$ \\
\hline VTE & 46.75 \\
\hline
\end{tabular}

Nota: $* p=.000$. Procedimientos realizados por el método de ejes principales y rotación promax, Kappa $=4$ (Osborne, 2014). 


\section{Análisis confirmatorio}

Para este análisis se evaluó la normalidad de los siete ítems de la MARS-A, hallándose datos de simetría que oscilaron entre -1.990 y -.022 y de curtosis de entre -1.631 y 3.155 , lo que confirma la no normalidad de los datos; con base en esto se empleó el método de máxima verosimilitud con corrección robusta de Browne para evaluar la bondad de ajuste del modelo, encontrándose que la MARS-A cuenta con un coeficiente $\alpha$ de .850 , las saturaciones de los ítems oscilan entre $.42 \mathrm{y}$ .76, y los datos para los índices de ajuste absoluto muestran -previa unión de los errores $1 \leftrightarrow 2$ y $5 \leftrightarrow 9-$, una prueba de bondad de ajuste $X^{2}$ de 20,356 ( $p=.061)$, con $12 \mathrm{gl}$, determina un buen ajuste; el cociente $X^{2} / g l=1.69$ y el RMSEA de .062 establecen un ajuste aceptable, y el RMR de .046 indica un buen ajuste. Respecto a los índices de ajuste general, se encontró que el GFI de .971 y el AGFI de .933 fijaron un buen ajuste. Los índices de ajuste comparativo TLI de .963 y el NFI de .951 corroboran satisfacción en el modelo planteado. Finalmente, se obtuvo un índice AVE de 0.80501, lo que ratifica la existencia de adecuación convergente, ya que más de $50 \%$ de la varianza del constructo está en función de sus indicadores.

\section{Calificación de la MARS-A}

La calificación de la escala se obtiene sumando las respuestas de cada ítem (rango 7-28) y dividiendo esa suma entre el número de ítems, lo que implica que a mayor puntaje, mejor nivel de adherencia. Tomando como base la forma de interpretación de la escala original y considerando los ajustes realizados en el presente trabajo, se planteó un puntaje de 26 (3.7) para diferenciar a los pacientes adherentes de los no adherentes a un nivel de $90 \%$ de adherencia, porcentaje recomendado en los estudios sobre esta variable (Dunbar-Jacob et al., 2012; Stirratt et al., 2015).

\section{DISCUSIÓN}

Ya que el objetivo del presente trabajo fue evaluar las características psicométricas de la MARS-A en adultos con asma, se puede concluir que la escala tiene propiedades psicométricas adecuadas para la evaluación de conductas de falta de adherencia en este tipo de pacientes debido a que, por un lado, la escala final cuenta con un coeficiente alfa de Cronbach de 0.850 , valor bastante adecuado para considerarla confiable; además, este tipo de evidencia se corresponde con los datos de la escala en la versión original, inglesa (Horne y Hankins, 2002) y las versiones en inglés y español validadas en Estados Unidos (Cohen et al., 2009; Mora et al., 2011). Asimismo, también se corresponde con las versiones adaptados en otros países para pacientes con dolor crónico (Sampaio et al., 2019), enfermedad de Crohn (Scribano et al., 2018) e infarto (Lin et al., 2018).

En lo que atañe a la estructura factorial, se determinó que es necesario considerar una escala unifactorial con siete ítems dadas las evidencia teóricas y visuales. En este sentido, las adaptaciones hechas en diferentes contextos (Alsous et al., 2017; Cohen et al., 2009; García et al., 2016; Ladova et al., 2014; Lin et al., 2018; Mahler et al., 2010; Sampaio et al., 2019; Scribano et al., 2018; Tommelein et al., 2014) se corresponden en su mayoría con la propuesta original unifactorial (Horne y Hankins, 2002), únicamente diferenciándose en el número de ítems (cinco, seis, nueve o diez) y el tipo de pacientes y medicamentos. Sin embargo, hay dos estudios en los que se reporta una estructura de dos factores: uno con pacientes con asma, denominado falta de adherencia, basado en cambios somáticos y falta de adherencia intencional (Mora et al., 2011), y otro con pacientes con artritis reumatoide (Salt et al., 2012). Este último presenta inconsistencias en sus datos ya que considera un factor con seis ítems (inconsistencia en adherencia) y otro con tres ítems (adherencia con base en síntomas), uno de ellos cargando de manera importante en ambos factores (.71 y .56), y finalmente tomando en cuenta que no es adecuado que un factor tenga dos o tres ítems o solamente dos (Ferrando y Anguiano, 2010; Reyes y García, 2008).

Siguiendo esta misma línea en lo que respecta a la evaluación de la validez estructural, es necesario señalar que la mayoría de los estudios emplean técnicas de factorización basadas en el análisis de componentes principales con rotación varimax (Alsous et al., 2017; Cohen et al., 2009; 
Horne y Hankins, 2002; Lin et al., 2018; Mahler et al., 2010; Sampaio et al., 2019), lo que se considera inadecuado en cuanto que este tipo de factorizaciones no se orientan a identificar una estructura factorial sino, por el contrario, a reducir el número de variables. Además, se sugiere la rotación empleada cuando se puede garantizar que los datos se distribuyen normalmente (Ferrando y Anguiano, 2010), lo que es poco probable en la distribución de los datos de la MAR-S en virtud de que, como ocurrió en el presente estudio, las respuestas de los pacientes muestran tendencia hacia las opciones 3 y 4 (puntajes altos), lo que se corrobora en la distribución de respuestas y en las medidas de tendencia central mostradas en los estudios revisados (Alsous et al., 2017; Ladova et al., 2014; Lin et al., 2018; Mahler et al., 2010; Sampaio et al., 2019; Scribano et al., 2018; Tommelein et al., 2014). Por el contrario, solamente el estudio de Salt et al. (2012) utilizó un análisis basado en ejes principales, el cual se recomienda cuando los datos no son normales (Osborne, 2014).

En el presente estudio se observó que, en el análisis factorial exploratorio, fueron dos ítems los que se eliminaron por no cumplir el criterio $\geq .4$ en la carga factorial; los cuales fueron el 7 ("Uso mi medicamento de control si mi tratamiento de rescate no funciona") y el 8 ("Uso mi medicamento de control antes de hacer algo que me provoque dificultad para respirar"). En los estudios de pacientes con asma de Horne y Hankins (2002) y Cohen et al. (2009) dichos ítems son parte de la dimensión Falta de adherencia intencional, pero en el estudio de Mora et al. (2011) se agrupan en el factor Falta de adherencia intencional basado en cambios somáticos experimentados, dado el contenido de los ítems. Sin embargo, en el presente estudio se ubicaron, por un lado, como los ítems con correlaciones más bajas con la escala (.415 y .262), así como las cargas factoriales más bajas en el análisis exploratorio (.357 y .247). Estos datos no resultan extraños si se considera que, de hecho, los pacientes con asma se comportarían al revés de como lo describe el ítem 7, esto es, normalmente usarán más el medicamento de rescate si el de control no funciona, lo cual es muy común ya que esa preferencia se basa en la rápida disminución de los síntomas, lo que no sucede con el medicamento de control (Halm, Mora y Leventhal,
2006; Ulrik et al., 2006). Esta forma de comportamiento, identificada como un uso excesivo del medicamento de rescate, implica otra conducta de falta de adherencia (Ebmeier et al., 2017; Engelkes et al., 2015; GINA, 2016; Rifaat, Abdel-Hadid y Hasan, 2013). Ahora bien, en lo referente al ítem 8 , se identifica una incompatibilidad con las recomendaciones hechas por los neumólogos, pues normalmente se recomienda el uso del medicamento de rescate, y no el de control, diez minutos antes de la realización de actividad física, siempre y cuando sea un paciente con asma inducida por ejercicio y presente síntomas de asma antes y después de realizar el mismo (GINA, 2016; GUIMA; 2017).

En relación con el análisis factorial confirmatorio, y considerando que Mora et al. (2011) propuso un análisis con base en modelamiento de ecuaciones estructurales en el que obtuvo los siguientes indicadores: $X^{2}=37.69, p=.05$, RMSEA $=.043$, $\mathrm{CFI}=.98$ y $\mathrm{TLI}=.96$, al contrastarlo con el modelo del presente estudio se hallan diferencias en algunos indicadores ( $X^{2}, p$ y RMSEA); sin embargo, se considera en general que ambos modelos muestran un buen ajuste.

Finalmente, y con base en los resultados obtenidos, se reitera que la MARS-A es una escala confiable y válida para la evaluación de conductas de falta de adherencia a medicamentos de control, de una manera breve y económica, dado el número de ítems con los que cuenta y el tiempo que se emplea en responderla; es además una herramienta centrada en el patrón de uso de los medicamentos, derivada de una definición conductual sobre la adherencia y que considera evitar el juicio de valor negativo o amenazante en sus instrucciones e ítems, características que deben indicarse en una herramienta de evaluación del autorreporte de adherencia (Dunbar-Jacob et al., 2012; Foster et al., 2011; Horne y Weinman, 1999; Lam y Fresco, 2015; Mora et al., 2011; Stirratt et al., 2015).

Es importante señalar que la forma de obtención de la muestra puede representar un problema en cuanto que fue de tipo no probabilístico y realizada en consulta externa, lo que podría considerar un perfil de pacientes con características con poca gravedad de la enfermedad o un tiempo prolongado con ella, lo que es una crítica frecuente reportada en los estudios de adherencia con pacientes 
con asma y la cual se recomienda evitar (Jessop y Rutter, 2003). No obstante, en la descripción de las muestras se puede observar cierta homogeneidad en diferentes variables, como el estado civil, la escolaridad, la ocupación y la severidad de la enfermedad, que muestran cierta variabilidad; no así en la constante de sexo debido a que la prevalencia de asma es mayor en mujeres que en hombres y durante la adolescencia y la adultez, al contrario de lo que ocurre en la niñez, donde la prevalencia es mayor entre los varones (Choo, Hoyte y Katial, 2014; GINA, 2016; GUIMA, 2017; Miller y Sawlani, 2013).
Finalmente, es necesario considerar que a pesar de que la MARS-A muestra las diversas bondades previamente mencionadas, no se pueden obviar los errores en la medición, la sobrestimación que hacen los pacientes y la deseabilidad social que se aprecia en sus respuestas, dado el tipo de comportamiento evaluado y el lugar en el que tal evaluación se efectúa. Como un indicador de este problema se reitera la tendencia de las respuestas identificada en los diversos estudios ya mencionados.

\section{REFERENCIAS}

Alsous, M., Alhalaiqa, F., Abu Farha, R., Abdel, J.M., McElnay, J. y Horne, R (2017). Reliability and validity of Arabic translation of Medication Adherence Report Scale (MARS) and Beliefs about Medication Questionnaire (BMQ) \pm specific for use in children and their parents. PLOS ONE, 12(2). 1-14. doi:10.1371/journal.pone.0171863.

Bender, B.G. y Bender, S.E. (2005). Patient-identified barriers to asthma treatment adherence: responses to interviews, focus groups, and questionnaires. Immunology and Allergy Clinics of North America, 25(1), 107-130. doi.org/10.1016/j.iac.2004.09.005.

Browne, M.W. y Cudeck, R. (1993). Alternative ways of assessing model fit. Sage Focus Editions, 154, 136-136. doi: $10.1177 / 0049124192021002005$.

Chapman, S.C., Barnes, N., Barnes, M., Wilkinson, A., Hartley, J., Piddock, C., Weinman, J. y Horne, R. (2015). Changing adherence-related beliefs about ICS maintenance treatment for asthma: feasibility study of an intervention delivered by asthma nurse specialists. British Medical Journal Open, 5(6), 1-9. doi: 10.1136/bmjopen-2014-007354.

Choo, E.M., Hoyte, F.C.L. y Katial, R.K. (2014). Adult and pediatric asthma. En P. K Vedanthan, H. Nelson, S. H. Agashe, P. A. Mahesh y R. Katial (Eds.): Textbook of allergy for the clinician (pp. 135-142). Boca Raton, FLO: CRC Press Taylor \& Francis Group.

Cohen, J.L., Mann, D.M., Wisnivesky, J.P., Horne, R., Leventhal, H., Musumeci-Szabó, T. J. y Halm, E.A. (2009). Assessing the validity of self-reported medication adherence among inner-city asthmatic adults: The Medication Adherence Report Scale for Asthma. Annals of Allergy, Asthma y Immunology, 103(4), 325-331. doi: org/10.1016/S1081-1206(10)60532-7.

DeVellis, R.F. (2012). Scale development: theory and applications. Thousand Oaks, CA: Sage Publications.

DiMatteo, M.R., Haskard-Zolnierek, K.B. y Martin, L.R. (2012). Improving patient adherence: a three-factor model to guide practice. Health Psychology Review, 6(1), 74-91. doi: 10.1080/17437199.2010.537592.

Dirección General de Epidemiología (2015). Anuarios de morbilidad 1984-2015. México: Secretaría de Salud. Recuperado de http://www.epidemiologia.salud.gob.mx/anuario/html/anuarios.html.

Dunbar-Jacob, J., Schlenk, E. y McCall, M. (2012). Patient adherence to treatment regimen. En A. Baum, T. A. Revenson y J. Singer (Eds.): Handbook of Health Psychology (2nd ed.) (pp. 271-292). London: Routledge. Taylor \& Francis Books.

Ebmeier, S., Thayabaran, D., Braithwaite, I., Bénamara, C., Weatherall, M. y Beasley, R. (2017). Trends in international asthma mortality: analysis of data from the WHO Mortality Database from 46 countries (1993-2012). The Lancet, 390, $935-945$.

Emilsson, M., Berndtsson, I., Lötvall, J., Millqvist, E., Lundgren, J., Johansson, A. y Brink, E. (2011). The influence of personality traits and beliefs about medicines on adherence to asthma treatment. Primary Care Respiratory Journal, 20(2), $141-147$. doi: $10.4104 /$ pcrj.2011.00005.

Engelkes, M., Janssens, H.M., de Jongste, J.C., Sturkenboom, M.C. y Verhamme, K.M. (2015). Medication adherence and the risk of severe asthma exacerbations: a systematic review. European Respiratory Journal, 45(2), 396-407. doi: $10.1183 / 09031936.00075614$.

Farrell, A.M. (2010). Insufficient discriminant validity: A comment on Bove, Pervan, Beatty, and Shiu (2009). Journal of Business Research, 63(3), 324-327. doi: 10.1016/j.jbusres.2009.05.003.

Fernández R., C. y Merino S., R. (2015). La adhesión a los tratamientos médicos: concepto, medida y factores implicados. En V. Plaza y C. Fernández (Eds.): Adhesión terapéutica en las enfermedades respiratorias (pp 1-12). Barcelona: Viguera Editores. 
Ferrando P., J. y Anguiano C., C.(2010). El análisis factorial como técnica de investigación en psicología. Papeles del Psicólogo, 31(1), 18-23.

Foster, J.M., Lavoide, K.L. y Boulet, L.P. (2011). Treatment adherence and psychosocial factors in severe asthma. En K. F. Chung, E. H. Bel y S. E. Wenzel (Eds): European Respiratory Monograph: Dificult to treat severe asthma (pp. 28-49). European Respiratory Society. Lausanne: ERS. doi: 10.1183/1025448x.erm5110.

García, C., Fernández, R., Martínez, D., Franco, F. y Pérez, J. (2012). Prevalencia y riesgos asociados con pacientes adultos con asma de 40 años o más de la Ciudad de México: estudio de base poblacional. Salud Pública de México, 54, 425-432.

García C., B. y Pérez de Llano, L. (2015). Cumplimiento terapéutico y enfermedad pulmonar obstructiva crónica. Prevalencia, características y consecuencias. En V. Plaza y C. Fernández (Eds.): Adhesión terapéutica en las enfermedades respiratorias. (pp. 45-58). Barcelona: Viguera Eds. S.L.U. https://www.researchgate.net/publication/299126211_.

Garcia M., P.W., Brand, P.L., Kaptein, A.A. y Klok, T. (2016). Is the MARS questionnaire a reliable measure of medication adherence in childhood asthma? Journal of Asthma, 53(10), 1085-1089. doi: 10.1080/02770903.2016.1180699.

Global Initiative for Asthma (2016). Global strategy for asthma management and prevention. Fontana, WI: GINA.

Guía Mexicana de Asma (2017). Neumología y Cirugía de Tórax, 76(S1), 1-137.

Halm, E., Mora, P. y Leventhal, H. (2006). No Symptoms, no asthma. The acute episodic disease beliefs is associated with poor self-management among inner-city adults with persistent asthma. Chest, 129, 573-580. doi: 10.1378/chest.129.3.573.

Hernández S., R., Fernández C., C. y Baptista L., P. (2014). Metodología de la investigación. México: McGraw-Hill.

Horne, R. (2011). Adherence to asthma medication: a question of ability? Primary Care Respiratory Journal, 20(2), 118-119. doi: 118-119. DOI: $10.4104 /$ pcrj.2011.00054.

Horne, R. y Hankins, M. (2002). The Medication Adherence Report Scale. Brighton, UK: Center for Health Care Research.

Horne, R. y Weinman, J. (1999). Patients' beliefs about prescribed medicines and their role in adherence to treatment in chronic physical illness. Journal of Psychosomatic Research, 47(6), 555-567. doi: 10.1016/S0022-3999(99)00057-4.

Horne, R. y Weinman, J. (2002). Self-regulation and self-management in asthma: Exploring the role of illness perceptions and treatment beliefs in explaining non-adherence to preventer medication. Psychology \& Health, 17(1), 17-32. doi: $10.1080 / 08870440290001502$.

Instituto Nacional de Enfermedades Respiratorias (2018). Informe anual 2017. México: INER.

Jessop, D.C. y Rutter, D.R. (2003). Adherence to asthma medication: The role of illness representations. Psychology and Health, 18(5), 595-612. doi: 10.1080/0887044031000097009.

Kline, R. (2011). Principles and practice of structural equation modeling. New York; The Guilford Press.

Ladova, K., Matoulkova, P., Zadak, Z., Macek, K., Vyroubal, P., Vlcek, J. y Morisky, D.E. (2014). Self-reported adherence by MARS-CZ reflects LDL cholesterol goal achievement among statin users: validation study in the Czech Republic. Journal of Evaluation in Clinical Practice, 20(5), 671-677. doi: 10.1111/jep.12201.

Laforest, L., El Hasnaoui, A., Pribil, C., Ritleng, C., Osman, L.M., Schwalm, M.S., Le Jeunne, P. y Van Ganse, E. (2009). Asthma patients' self-reported behaviours toward inhaled corticosteroids. Respiratory Medicine, 103(9), 1366-1375. doi:10.1016/j. rmed.2009.03.010.

Lam, W.Y. y Fresco, P. (2015). Medication adherence measures: an overview. BioMed Research International, 2015, 1-12.DOI: org/10.1155/2015/217047.

Lin, C.Y., Ou, H.T., Nikoobakht, M., Broström, A., Årestedt, K. y Pakpour, A.H. (2018). Validation of the 5-item Medication Adherence Report Scale in older stroke patients in Iran. Journal of Cardiovascular Nursing, 33(6), 536-543. doi: 10.1097/ JCN.0000000000000488.

López R., L.A., Romero G., S.L., Parra D., I. y Rojas S., L.Z. (2016). Adherencia al tratamiento: concepto y medición. Revista Hacia la Promoción de la Salud, 21(1), 117-137. doi: 10.17151/hpsal.2016.21.1.10.

Mahler, C., Hermann, K., Horne, R., Ludt, S., Haefeli, W.E., Szecsenyi, J. y Jank, S. (2010). Assessing reported adherence to pharmacological treatment recommendations. Translation and evaluation of the Medication Adherence Report Scale (MARS) in Germany. Journal of Evaluation in Clinical Practice, 16(3), 574-579. doi: 10.1111/j.1365-2753.2009.01169.x.

Menckeberg, T.T., Bouvy, M.L., Bracke, M., Kaptein, A.A., Leufkens, H.G., Raaijmakers, J.A. y Horne, R. (2008). Beliefs about medicines predict refill adherence to inhaled corticosteroids. Journal of Psychosomatic Research, 64(1), 47-54. doi: 10.1016/j.jpsychores.2007.07.016.

Méndez, I., Namihira, D., Moreno, L. y Sosa, C. (2001). El protocolo de investigación. Lineamientos para su elaboración y análisis. México: Trillas.

Miller, N. y Sawlani, S. (2013). Asma. En S. Joo, A. L. Kau, T. M. De Fer y K. E. Henderson (Eds.): The Washington ${ }^{\circledR}$ Manual for allergy and asthma (2nd ed.). Philadelphia, PA: Lippincott Williams \& Wilkins.

Mora, P.A., Berkowitz, A., Contrada, R.J., Wisnivesky, J., Horne, R., Leventhal, H. y Halm, E.A. (2011). Factor structure and longitudinal invariance of the Medical Adherence Report Scale-Asthma. Psychology \& Health, 26(6), 713-727. doi: 10.1080/08870446.2010.490585. 
Morata, R.M., Holgado T., F.P., Barbero G., I. y Méndez, G. (2015). Análisis factorial confirmatorio: recomendaciones sobre mínimos cuadrados no ponderados en función del error tipo I de Ji-Cuadrado y RMSEA. Acción Psicológica, 12(1), 79-90. Recuperado de http://scielo.isciii.es/pdf/acp/v12n1/articulo8.pdf.

Nunnally, J.C. y Bernstein, I.H. (1994). Psychometric theory. New York: McGraw-Hill.

Ohm, R. y Aaronson, L.S. (2006). Symptom perception and adherence to asthma controller medications. Journal of Nursing Scholarship, 38(3), 292-297. doi: 10.1111/j.1547-5069.2006.00116.x.

Osborne, J.W. (2014). Best practices in exploratory factor analysis. Scotts Valley, CA: CreateSpace.

Ovchinikova, L., Smith, L. y Bosnic-Anticevich, S. (2011). Inhaler technique maintenance: gaining an understanding from the patient's perspective. Journal of Asthma, 48(6), 616-624. doi: 10.3109/02770903.2011.580032.

Pedrosa, I., Juarros, J., Robles, A., Basteiro, J. y García, E. (2015). Pruebas de bondad de ajuste en distribuciones simétricas ¿qué estadístico utilizar? Universitas Psychologica, 14(1), 245. doi: 10.11144/Javeriana.upsy14-1.pbad.

Ponieman, D., Wisnivesky, J.P., Leventhal, H., Musumeci-Szabó, T.J. y Halm, E.A. (2009). Impact of positive and negative beliefs about inhaled corticosteroids on adherence in inner-city asthmatic patients. Annals of Allergy, Asthma \& Immunology, 103(1), 38-42. doi: 10.1016/S1081-1206(10)60141-X.

Reyes L., I. (1993). Las redes semánticas naturales, su conceptualización y su utilización en la construcción de instrumentos. Revista de Psicología Social y Personalidad, 9(1), 81-97. http://www.amepso.org/revistas.php.

Reyes L., I. (2011). Conceptualización y desarrollo de la etnopsicometría en México. En A. Domínguez (Comp.): Letras introductorias a la psicología cultural, transcultural y etnopsicología (pp. 279-302). México: Universidad Iberoamericana.

Reyes L., I. y García B., L. (2008). Procedimiento de validación psicométrica culturalmente relevante: un ejemplo. La Psicología Social en México, XII, 625-630.

Rifaat, N., Abdel-Hadid, E. y Hasan, A.A. (2013). The golden factor in adherence to inhaled corticosteroid in asthma patients. Egyptian Journal of Chest Diseases and Tuberculosis, 62, 371-376. doi: 10.1016/j.ejcdt.2013.07.010.

Rodríguez C., M.D.L. y Rentería, R.A. (2016). Factores que impiden la adherencia a un régimen terapéutico en diabéticos: un análisis descriptivo. Psicología y Salud, 26(1). http://revistas.uv.mx/index.php/psicysalud/article/view/1898.

Salt, E., Hall, L., Peden, A.R. y Horne, R. (2012). Psychometric properties of three medication adherence scales in patients with rheumatoid arthritis. Journal of Nursing Measurement, 20(1), 59-72. doi: 10.1891/1061-3749.20.1.59.

Sampaio, R., Azevedo, L.F., Dias, C.C., Horne, R. y Castro, C.J.M. (2019). Portuguese version of the Medication Adherence Report Scale (MARS-9): Validation in a population of chronic pain patients. Journal of Evaluation in Clinical Practice, 1-7. doi: 10.1111/jep.13098.

Scribano, M.L., Caprioli, F., Michielan, A., Contaldo, A., Privitera, A.C., Bozzi, R.M., Calabrese, E., Castiglione, F., Ciccaglione, A.F., Fave, G.D., Bodini, G., Costantino, G., Horne, H., Saettone, S., Usai, P., Vernia, P., Di Fino, S., Gualberti, G., di Fonzo, M., Merolla, R., Orlando, A. y The sole Study Group (2018). Translation and initial validation of the Medication Adherence Report Scale (MARS) in Italian patients with Crohn's Disease. Digestive and Liver Disease. doi: https://doi. org/10.1016/j.dld.2018.09.026.

Secretaría de Salud (2016). Siete por ciento de la población en México padece asma. Recuperado de: https://www.gob.mx/salud/ prensa/siete-por-ciento-de-la-poblacion-en-mexico-padece-asma.

Sofianou, A., Martynenko, M., Wolf, M., Wisnivesky, J., Krauskopf, K., Wilson, E., Sanghavi, M., Leventhal, H., Halm, M. y Federman, A. (2013). Asthma beliefs are associated with medication adherence in older asthmatics. Journal of General Internal Medicine, 28(1), 67-73. doi: 10.1007/s11606-012-2160-z.

Stirratt, M.J., Dunbar-Jacob, J., Crane, H.M., Simoni, J.M., Czajkowski, S., Hilliard, M.E., Aikens, J.E., Hunter, C.M., Velligan, D.I., Huntley, K., Ogedegbe, G., Rand, C., Schron, E. y Nilsen, W.J. (2015). Self-report measures of medication adherence behavior: recommendations on optimal use. Translational Behavioral Medicine, 5(4), 470-482. DOI: 10.1007/s13142-0150315-2.

Tommelein, E., Mehuys, E., Van Tongelen, I., Brusselle, G.y Boussery, K. (2014). Accuracy of the Medication Adherence Report Scale (MARS-5) as a quantitative measure of adherence to inhalation medication in patients with COPD. Annals of Pharmacotherapy, 48(5), 589-595. doi: 10.1177/1060028014522982.

Ulrik, C.S., Backer, V., Søes-Petersen, U., Lange, P., Harving, H.y Plaschke, P.P. (2006). The patient's perspective: adherence or non-adherence to asthma controller therapy? Journal of Asthma, 43(9), 701-704. doi: 10.1080/02770900600925569.

World Health Organization (2003). Adherence to long-term therapies: Evidence for action. Geneve: WHO. Recuperado de http:// www.who.int/chp/knowledge/publications/adherence_report/en/.

World Health Organization (2011). Asthma. Geneve: WHO.

Wroe, A.L. (2002). Intentional and unintentional nonadherence: a study of decision making. Journal of Behavioral Medicine, 25(4), 355-372. doi: 10.1023/A:1015866415552.

Zwikker, H.E., van den Ende, C.H., van Lankveld, W.G., den Broeder, A.A., van den Hoogen, F.H., van de Mosselaar, B., van Dulmen, S. y van den Bemt, B.J. (2014). Effectiveness of a group-based intervention to change medication beliefs and improve medication adherence in patients with rheumatoid arthritis: a randomized controlled trial. Patient Education and Counseling, 94(3), 356-361. doi: 10.1016/j.pec.2013.12.002. 